\title{
Risk factors for acute toxoplasmosis in England and Wales
}

\author{
B. SAID ${ }^{1}$, K. D. HALSBY ${ }^{1 *}$, C. M. O'CONNOR ${ }^{1}$, J. FRANCIS ${ }^{2}$, K. HEWITT ${ }^{3}$, \\ N. Q. VERLANDER ${ }^{4}$, E. GUY ${ }^{2}$ AND D. MORGAN ${ }^{1}$ \\ ${ }^{1}$ Emerging Infections and Zoonoses Section, National Infections Service, Public Health England, UK \\ ${ }^{2}$ Toxoplasma Reference Unit, Public Health Wales, UK \\ ${ }^{3}$ London Deanery, UK \\ ${ }^{4}$ Statistics, Modelling and Economics Department, National Infections Service, Public Health England, UK
}

Received 14 June 2016; Final revision 16 August 2016; Accepted 6 September 2016;

first published online 28 September 2016

\section{SUMMARY}

Over 300 cases of acute toxoplasmosis are confirmed by reference testing in England and Wales annually. We conducted a case-control study to identify risk factors for Toxoplasma gondii infection to inform prevention strategies. Twenty-eight cases and 27 seronegative controls participated. We compared their food history and environmental exposures using logistic regression to calculate odds ratios (OR) and 95\% confidence intervals in a model controlling for age and sex. Univariable analysis showed that the odds of eating beef (OR 10.7, $P<0.001$ ), poultry (OR 6.4, $P=0.01$ ) or lamb/mutton (OR 4.9, $P=0.01$ ) was higher for cases than controls. After adjustment for potential confounders a strong association between beef and infection remained (OR 5·6, $P=0 \cdot 01$ ). The small sample size was a significant limitation and larger studies are needed to fully investigate potential risk factors. The study findings emphasize the need to ensure food is thoroughly cooked and handled hygienically, especially for those in vulnerable groups.

Key words: Epidemiology, toxoplasmosis.

\section{INTRODUCTION}

Toxoplasmosis is a zoonotic disease caused by the protozoan parasite Toxoplasma gondii, which usually causes a mild illness in humans. However, toxoplasmosis can have severe implications for pregnant women or immunocompromised people. About $80 \%$ of acute Toxoplasma infections in immunocompetent hosts are asymptomatic [1]. When symptomatic infection occurs in immunocompetent individuals, common manifestations include lymphadenopathy and/

\footnotetext{
* Author for correspondence: Dr K. D. Halsby, Emerging and Zoonotic Infections Section, National Infections Service, Public Health England, NW9 5EQ, UK. (Email: kate.halsby@phe.gov.uk)
}

or influenza-like illness. Chorioretinitis can also occur, although more rarely [2]. Some epidemiological studies suggest a relationship between toxoplasmosis and the development of psychiatric disorders including schizophrenia [3]. In the immunocompromised, complications can include ocular or cerebral infection. In pregnant women, toxoplasmosis can result in miscarriage, stillbirth, or congenital toxoplasmosis in the baby, causing a range of potential long-term conditions.

Toxoplasma gondii can be found in the faeces of infected cats, in environments contaminated by infected cat faeces, and in the meat of infected animals such as pigs, cattle and sheep. In cats, the parasite infects and reproduces in the gut epithelial cells and 
oocysts are then excreted in faeces. In non-felines, including humans, ingestion of Toxoplasma oocysts can result in the organisms being disseminated throughout the body in the tachyzoite form during a parasitaemic phase. These tachyzoites can form intracellular tissue cysts within which they may remain in their bradyzoite form for the life of the host. These tissue cysts are infectious if consumed by other animals including humans [4].

Toxoplasmosis can be acquired in humans by: (i) ingesting sporulated oocysts from the environment, usually from soil, fruit or vegetables, or from water contaminated with feline faeces; (ii) ingesting cysts in food produced from an infected animal; (iii) vertical transmission from an infected mother to her fetus; (iv) blood transfusion or organ transplantation; and (v) ingesting the parasite following direct contact with products of conception during lambing [1, 4-8]. The relative role of consumption of cysts in undercooked meat products and intake of oocysts from soil or water contaminated by cat faeces is not known [9].

Toxoplasmosis is not a notifiable disease or organism in England and Wales. The current enhanced surveillance programme relies on identification of cases referred for specialist testing at the Public Health Wales Toxoplasma Reference Unit (TRU), where 300-450 cases are reported each year [10]. However, seroprevalence studies suggest that toxoplasmosis is significantly underreported [9].

Currently there are no national guidelines for the prevention of toxoplasmosis in England and Wales. Until fairly recently, infection with $T$. gondii was presumed to be primarily related to cat contact. A number of European studies in the 1990s (as detailed in the Discussion) identified associations with meat consumption in continental Europe, but uncertainty has remained as to whether British eating habits are sufficiently different to result in differences in the primary modes of transmission in the UK. The Advisory Committee on the Microbiological Safety of Food has acknowledged that the relative contribution of food as a route of transmission of Toxoplasma infection in the UK is not known, and has recommended that a case-control study be conducted to investigate the importance of food and environmental risk factors [9]. The European Food Safety Authority has also concluded that the relative roles of the potential routes of infection are unknown [1]. In order to maximize the effectiveness of any intervention strategy, a clear understanding is required of the relative importance and frequency of each of these modes of transmission.

This study therefore aims to investigate the potential risk factors in the UK in order to better inform possible prevention strategies. The study also aims to assess the feasibility of conducting a larger study using this methodology.

\section{METHODS}

Data on clinical cases of Toxoplasma infection in England and Wales are collected through an enhanced surveillance system delivered by Public Health England (PHE) and TRU. Laboratories refer samples to TRU for specialist diagnosis or reference testing when routine laboratory testing is not sufficient to achieve a definitive diagnosis or to fully inform clinical management. A substantial proportion of such samples come from vulnerable clinical groups.

TRU has developed a series of laboratory investigation pathways for the various clinical scenarios where specialist testing is required. Laboratory tests undertaken include the 'gold standard' Sabin-Feldman dye test, enzyme immunoassay (EIA) for the detection of specific IgG and IgM antibodies, immunosorbent agglutination assay for IgA or IgM, IgG avidity measurement, immunoblotting, histological examination of solid tissues, and nucleic acid amplification testing (real-time PCR). An acute case of Toxoplasma infection in an immunocompetent individual is defined, for the purposes of this study, as a patient presenting with (i) dye test $\geqslant 500 \mathrm{IU} / \mathrm{ml}$, and (ii) IgM levels $\geqslant 60 \mathrm{U} / \mathrm{ml}$ as measured by the TRU IgM EIA.

All adults confirmed as an acute case of Toxoplasma infection by TRU between December 2012 and December 2013 were contacted by post and invited to participate in the study. The laboratories that referred positive cases to TRU were asked to supply a list of individuals who had tested negative for $T$. gondii. These individuals were then contacted by post and invited to participate as controls. If no response was received, a second and final invitation letter was sent.

Each person who agreed to take part in the study was contacted by telephone and interviewed using a structured questionnaire. At least three attempts at telephone contact were made. Pregnant individuals were excluded because they might be following pregnancy-associated dietary recommendations, and on ethical grounds due to potential distress. Additionally, individuals who had travelled abroad 
in the month before onset of symptoms (or the date of interview for controls) were excluded since the study aimed to examine the risk of infection in the UK only. The questionnaire gathered demographic and clinical information, and details of the foods that they were likely to have eaten in the month before onset of symptoms (cases) or interview (controls). Sections on different food groups (e.g. beef, pork, lamb) included questions on how it would have been prepared. Participants were asked about other potential exposures such as contact with cats, type of housing and leisure activities. For each case, we attempted to recruit two controls matched by reporting laboratory, and assuming a response rate of $25 \%$ this meant that we had a required sample size of 225 .

Data were stored in a secure password-protected database, and were cleaned and analysed using Stata/SE v. 13.1 (StataCorp, USA). Logistic regression was used to calculate odds ratios (ORs) to examine associations between potential risk factors and cases of Toxoplasma in a univariable analysis. We developed a multivariable logistic regression model to obtain adjusted ORs and their 95\% confidence intervals (CIs). Exposures which were identified as potential risk factors in a univariable analysis (OR $>1$ and $P \leqslant 0 \cdot 2$ ) and to which $>30 \%$ of cases were exposed, were included in the model and adjusted ORs and their 95\% CIs calculated. Age and sex were included in the model, with linearity of age with outcome on the logit scale tested. Any food item with a statistically significant association with case status in the multivariable model was explored further by replacing it in the model with a variable indicating how thoroughly the food had been cooked. $P$ values were calculated using the likelihood ratio test and statistical significance was set at $5 \%$. In those instances where an OR could not be estimated due to sampling zeros, exact logistic regression was used to obtain a median unbiased estimate of the OR.

Ethical approval for this study was granted by the National Information Governance Board for Health and Social Care.

\section{RESULTS}

Of 438 referrals received at TRU from laboratories between December 2012 and December 2013, 55 participants (28 cases and 27 controls) completed questionnaires for the study (response rate 12.6\%; Fig. 1). The response rate was low and it was not possible to match participants by laboratory, a limitation highlighted further in the Discussion.

Thirty-five $(63.6 \%)$ participants were female and 52 (94.5\%) gave their ethnicity as 'white', with no difference between cases and controls. Controls were slightly older than cases (median age of controls 46 years, median age of cases 37.5 years).

A third of controls $(33 \cdot 3 \%)$ were immunocompromised compared to $17.9 \%$ of cases. When the country was divided into Government Office Regions [11], there was at least one case and control reported from each region, with the majority from the South of England (12 cases, 15 controls).

Data completion was very high, as might be expected from telephone interviews. Three exposure variables had one answer missing each (all from different individuals), and the 'preparation of beef' variable had eight missing values.

In the univariable analysis, cases had a higher odds of having eaten beef (OR 10·7, 95\% CI 1.49- $\infty, P=0 \cdot 001$ ), lamb/mutton (OR 1.3, 95\% CI 1.29-18.8, $P=0 \cdot 01$ ) or poultry (OR $6 \cdot 3,95 \%$ CI $0.74-\infty, P=0 \cdot 01$ ) (Table 1 ). None of the other food exposures or environmental risk factors, such as contact with cats or cat ownership, showed an association at the 5\% significance level.

The variables entered into the multivariable model (with $>30 \%$ of cases exposed and where OR $>1$ and $P \leqslant 0 \cdot 2$ ) were: beef, pork, lamb/mutton, poultry, dairy, and gardening. Eating BBQ and hunting both had $P$ values $<0 \cdot 2$, but $<30 \%$ of cases were exposed. After adjustment for the other variables of interest, only beef remained associated with infection (OR $5 \cdot 6,95 \%$ CI $0 \cdot 63-\infty, P=0 \cdot 01$ ) (Table 2). All CIs for this model crossed 1 .

The associations were further explored by replacing the food item 'beef' in the model with a variable indicating how thoroughly the food had been cooked ('preparation type'). There was a significant improvement in model fit compared to a model without any beef variable $(P<0 \cdot 0007)$. The largest $\mathrm{OR}$ was returned for the category 'raw to medium rare' (OR $5 \cdot 8$ ) compared to 'well done' beef (Table 3).

\section{DISCUSSION}

Risk factors for toxoplasmosis in England and Wales are poorly described. This study suggests an association between consumption of beef and toxoplasmosis, especially when the beef is cooked rare or medium rare.

These findings are consistent with other European studies that found evidence of an association between 


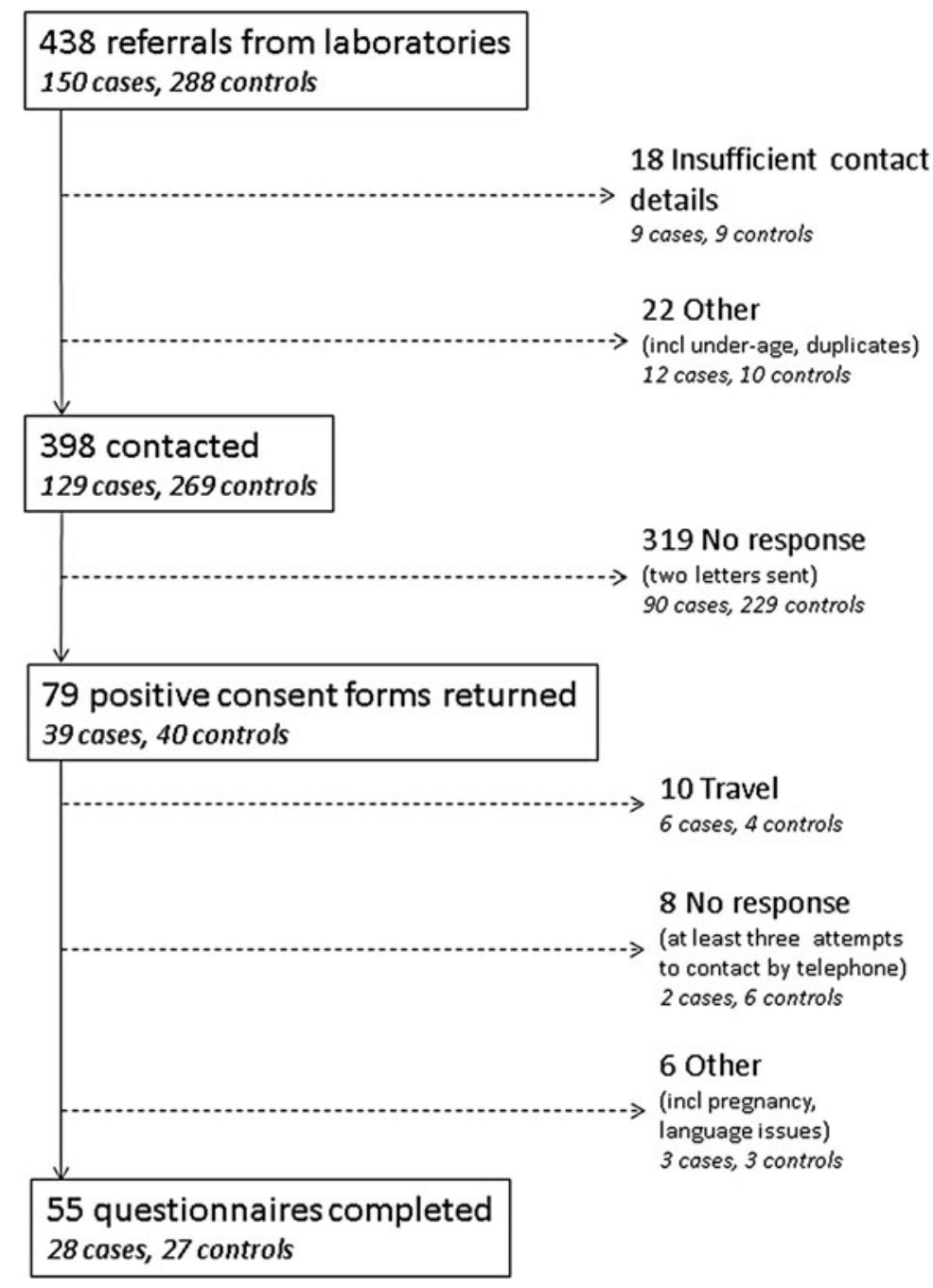

Fig. 1. Case and control exclusions and losses.

raw or undercooked meat and Toxoplasma infection in humans. While the overall finding is relatively consistent between studies, the types of meat posing the greatest risk are variable. Cook et al. conducted a European multi-centre study in pregnant women where the risk factors most strongly predictive of acute infection were eating undercooked lamb, beef or game, contact with soil, and travel outside Europe and the United States and Canada [12]. Buffolano et al. found an association between recent infection in pregnant women in Italy and consumption of cured pork and raw meat (of unspecified type) [13]. Kapperud et al. discovered associations between increased risk of maternal infection and a large number of factors including (among others) undercooked minced meat products, undercooked mutton, undercooked pork and cleaning cat litter boxes [5]. Baril et al. investigated Toxoplasma infection in pregnant women in France and found that consumption of undercooked beef, consumption of raw vegetables outside the home, and having a pet cat were significantly associated with Toxoplasma infection [14].

While these European studies have suggested for some time that meat is an important source of $T$. gondii infection, food preferences and dietary habits in the UK have been considered sufficiently distinct, that the relative importance of potential risk factors may be different. Our study has demonstrated that this is not the case, and suggests that undercooked meat is an important risk in England and Wales, as it is across the rest of Europe.

There has been one previous study examining the risk factors for $T$. gondii infection in the UK. Nash et al. examined a cohort of pregnant women who were tested for Toxoplasma antibodies, and found evidence that feeding a dog raw meat, living on a farm or in the countryside as a child, living in mainland 
Table 1. Univariable analysis, after adjusting for age and sex (in all analyses, a linear association on the logit scale of age with outcome was found to be adequate)

\begin{tabular}{|c|c|c|c|c|c|c|c|}
\hline Food & & Controls & Cases & $\begin{array}{l}\text { Proportion of cases } \\
\text { exposed }\end{array}$ & OR & $95 \% \mathrm{CI}$ & $P$ value \\
\hline \multirow[t]{2}{*}{ Ate beef } & Yes & 20 & 28 & $100 \%$ & $10 \cdot 7 *$ & $1 \cdot 49-\infty$ & $0 \cdot 001$ \\
\hline & No & 7 & 0 & & & & \\
\hline \multirow[t]{2}{*}{ Ate poultry } & Yes & 23 & 28 & $100 \%$ & $6 \cdot 34^{*}$ & $0 \cdot 74-\infty$ & $0 \cdot 01$ \\
\hline & No & 4 & 0 & & & & \\
\hline \multirow[t]{2}{*}{ Ate lamb/mutton } & Yes & 15 & 22 & $79 \%$ & 4.93 & $1 \cdot 29-18 \cdot 8$ & $0 \cdot 01$ \\
\hline & No & 12 & 6 & & & & \\
\hline \multirow[t]{2}{*}{ Ate dairy } & Yes & 25 & 28 & $100 \%$ & $3 \cdot 47^{*}$ & $0 \cdot 26-\infty$ & $0 \cdot 05$ \\
\hline & No & 2 & 0 & & & & \\
\hline \multirow[t]{2}{*}{ Ate egg } & Yes & 24 & 27 & $96 \%$ & $3 \cdot 46$ & $0 \cdot 32-37 \cdot 5$ & $0 \cdot 28$ \\
\hline & No & 3 & 1 & & & & \\
\hline \multirow[t]{2}{*}{ Ate BBQ } & Yes & 3 & 6 & $21 \%$ & $2 \cdot 97$ & $0.57-15 \cdot 4$ & $0 \cdot 18$ \\
\hline & No & 24 & 22 & & & & \\
\hline \multirow[t]{2}{*}{ Ate pork } & Yes & 20 & 25 & $89 \%$ & $2 \cdot 70$ & $0 \cdot 60-12 \cdot 3$ & $0 \cdot 18$ \\
\hline & No & 7 & 3 & & & & \\
\hline \multirow[t]{2}{*}{ Gardening } & Yes & 14 & 17 & $61 \%$ & $2 \cdot 58$ & $0 \cdot 69-9 \cdot 65$ & $0 \cdot 15$ \\
\hline & No & 12 & 11 & & & & \\
\hline \multirow[t]{2}{*}{ Ate shellfish } & Yes & 22 & 25 & $89 \%$ & $2 \cdot 37$ & $0 \cdot 48-11 \cdot 7$ & $0 \cdot 28$ \\
\hline & No & 5 & 3 & & & & \\
\hline \multirow[t]{2}{*}{ Hunting } & Yes & 0 & 2 & $7 \%$ & $2 \cdot 12^{*}$ & $0 \cdot 16-\infty$ & $0 \cdot 11$ \\
\hline & No & 27 & 26 & & & & \\
\hline \multirow[t]{2}{*}{ Contact soil } & Yes & 5 & 6 & $21 \%$ & 1.83 & $0 \cdot 41-8 \cdot 19$ & $0 \cdot 43$ \\
\hline & No & 22 & 22 & & & & \\
\hline \multirow[t]{2}{*}{ Contact animals } & Yes & 4 & 5 & $18 \%$ & 1.72 & $0.37-8.09$ & $0 \cdot 49$ \\
\hline & No & 23 & 23 & & & & \\
\hline \multirow[t]{2}{*}{ Contact cat } & Yes & 9 & 12 & $43 \%$ & 1.65 & $0 \cdot 46-5 \cdot 91$ & $0 \cdot 44$ \\
\hline & No & 18 & 16 & & & & \\
\hline \multirow[t]{2}{*}{ Playground/parks } & Yes & 4 & 7 & $25 \%$ & 1.55 & $0 \cdot 38-6 \cdot 38$ & $0 \cdot 54$ \\
\hline & No & 22 & 21 & & & & \\
\hline \multirow[t]{2}{*}{ Hiking } & Yes & 10 & 13 & $46 \%$ & 1.52 & $0 \cdot 50-4 \cdot 59$ & $0 \cdot 46$ \\
\hline & No & 17 & 15 & & & & \\
\hline \multirow[t]{2}{*}{ Swim lakes, ponds, rivers } & Yes & 1 & 0 & $0 \%$ & $1 \cdot 24^{*}$ & $0-48 \cdot 5$ & $0 \cdot 27$ \\
\hline & No & 26 & 28 & & & & \\
\hline \multirow[t]{2}{*}{ Own cat } & Yes & 8 & 9 & $32 \%$ & $1 \cdot 00$ & $0 \cdot 28-3 \cdot 62$ & 1.00 \\
\hline & No & 19 & 19 & & & & \\
\hline \multirow[t]{2}{*}{ Swim in sea } & Yes & 5 & 4 & $14 \%$ & $0 \cdot 83$ & $0 \cdot 19-3 \cdot 62$ & $0 \cdot 81$ \\
\hline & No & 22 & 24 & & & & \\
\hline \multirow[t]{2}{*}{ Raw milk } & Yes & 1 & 1 & $4 \%$ & $0 \cdot 82$ & $0 \cdot 05-14 \cdot 8$ & 0.89 \\
\hline & No & 26 & 27 & & & & \\
\hline \multirow[t]{2}{*}{ Raw meat } & Yes & 0 & 1 & $4 \%$ & $0 \cdot 74 *$ & $0.02-\infty$ & $0 \cdot 28$ \\
\hline & No & 27 & 27 & & & & \\
\hline
\end{tabular}

OR, Odds ratio; CI, confidence interval.

* Median unbiased estimate.

Europe (excluding the UK) as a child, and those aged $\geqslant 35$ years were all significantly associated with seropositivity [15]. However, they found no evidence of significant dietary risk factors in this group and these authors suggested that this may be due to the limitations of the serological tests used to determine infection status, i.e. detection of $\operatorname{IgG}$ alone and in the absence of IgM detection could not discriminate between recent infection and infection acquired years or decades previously. Given that dietary habits may change significantly over such a potentially long period, the correlation between current risk factors and factors that applied at the time the infection was acquired may have been poor.

Care must be taken with the conclusions drawn from the data because the CIs for the adjusted OR 
Table 2. Multivariable model results, after adjusting for age and sex

\begin{tabular}{llll}
\hline \hline Food & OR & $95 \%$ CI & $P$ value \\
\hline Beef & $5 \cdot 59^{*}$ & $0 \cdot 63-\infty$ & $0 \cdot 01$ \\
Gardening & $3 \cdot 98$ & $0 \cdot 77-20 \cdot 5$ & $0 \cdot 09$ \\
Dairy & $3 \cdot 86^{*}$ & $0 \cdot 10-\infty$ & $0 \cdot 06$ \\
Lamb/mutton & $3 \cdot 07$ & $0 \cdot 62-15 \cdot 2$ & $0 \cdot 16$ \\
Poultry & $2 \cdot 81^{*}$ & $0 \cdot 07-\infty$ & $0 \cdot 08$ \\
Pork & $2 \cdot 77$ & $0 \cdot 33-23 \cdot 3$ & $0 \cdot 35$ \\
\hline \hline
\end{tabular}

OR, Odds ratio; CI, confidence interval.

* Median unbiased estimate.

Table 3. 'Preparation of beef' substituted for 'beef' in multivariable model (whose results are given in Table 2)

\begin{tabular}{lllll}
\hline \hline Preparation of beef & Control & Case & OR & $95 \%$ CI \\
\hline Beef not eaten & 7 & 0 & $0 \cdot 19 *$ & $0-2 \cdot 07$ \\
Raw to medium rare & 5 & 16 & $5 \cdot 76$ & $0 \cdot 49-68 \cdot 2$ \\
Medium & 8 & 6 & $0 \cdot 15$ & $0 \cdot 01-1 \cdot 80$ \\
Well done & 7 & 5 & Baseline & Baseline \\
\hline
\end{tabular}

OR, Odds ratio; CI, confidence interval.

* Median unbiased estimate.

in the multivariable regression models were very wide and crossed 1 . This is a significant limitation of the study and is likely to have been due to low power resulting from a small study size. This study used a combination of established methods of recruitment where potential participants were contacted first by post and, following their written agreement, subsequently by telephone $[16,17]$. However, in our study the uptake was poor and became a serious limiting factor. The study aimed to recruit two controls per case, matched on reporting laboratory. However, controls matched by laboratory proved to be unfeasible and even when the protocol was modified to remove this restriction, we were still unable to recruit one control per case. Requiring the controls to have tested negative for Toxoplasma was onerous; however, seropositivity for $T$. gondii infection is high in England and Wales, and it was important that controls had not had a Toxoplasma infection prior to participation in the study. While there was a higher response rate in cases than controls $(18 \cdot 7 \%$ vs. $9 \cdot 4 \%)$, most potential participants failed to respond at all. This may reflect changing attitudes to telephone surveys, and alternative methods of participation (e.g. internet survey, postal survey) should be considered for future studies.

The study methodology is based on food likely to have been consumed by the participant in the month before questionnaire for controls and the month before onset of symptoms for cases. While this may lead to a risk of recall bias, the study relied on food preferences, which remain consistent over time, rather than consumption, so that any recall bias was reduced.

T. gondii has been identified as the foodborne pathogen associated with the second highest public health impact both in the USA and The Netherlands $[18,19]$. Currently, in the UK there are no agreed national guidelines for prevention of toxoplasmosis. Both the life-cycle of the parasite and the modes of transmission provide opportunities for intervention and prevention. A clearer and more precise understanding of the relative importance of these will greatly inform the optimization of future guidelines for the UK population.

\section{CONCLUSIONS}

The results of the current study suggest an association between beef and toxoplasmosis, especially when the beef is cooked rare or medium rare. The association between undercooked beef and toxoplasmosis could be used as the basis for guidelines for the prevention of toxoplasmosis, especially in vulnerable groups such as pregnant women and the immunocompromised. This would be consistent with current recommendations that pregnant women ensure food is thoroughly cooked. Careful handling of raw and partially cooked meats is also advisable. The authors recommend that this work should be extended to a larger prospective study that minimizes recall bias and that future methodologies seek ways to maximize rates of recruitment.

\section{ACKNOWLEDGEMENTS}

The authors acknowledge the work of L. Pedrozzi, M.-A. Dugail, and H. Kirkbride in helping to develop the study protocols and obtaining ethics approval, B. Ben-Ismaeil for his assistance in conducting interviews, and J. McCormick for her help with data entry. We also like thank all of the collaborating laboratories and NHS trusts who gave permissions and provided data for the study.

\section{DECLARATION OF INTEREST}

None. 


\section{REFERENCES}

1. EFSA. Surveillance and monitoring of Toxoplasma in humans, food and animals (http://www.efsa.europa.eu/ sites/default/files/scientific_output/files/main_documents/ biohaz_op_ej583_toxoplasma_en\%2C3.pdf). EFSA Journal 2007; 583: 1-64.

2. McCabe R, et al. Clinical spectrum in 107 cases of toxoplasmic lymphadenopathy. Review of Infectious Diseases 1987; 9: 754-774.

3. Torrey E, Yolken R. Schizophrenia and toxoplasmosis. Schizophrenia Bulletin 2007; 33: 727-728.

4. Tenter A, Heckerroth A, Weiss L. Toxoplasma gondii: from animals to humans. International Journal of Parasitology 2000; 30: 1217-1258.

5. Kapperud G, et al. Risk factors for Toxoplasma gondii infection in pregnancy. Results of a prospective casecontrol study in Norway. American Journal of Epidemiology 1996; 144: 405-412.

6. Tenter A. Toxoplasma gondii in animals used for human consumption. Memórias do Instituto Oswaldo Cruz 2009; 104: 364-369.

7. Bowie $\mathbf{W}$, et al. Outbreak of toxoplasmosis associated with municipal drinking water. The BC Tocoplasma Investigation Team. Lancet 1997; 350: 173-177.

8. PHE. Toxoplasmosis: diagnosis, epidemiology and prevention. (https://www.gov.uk/guidance/toxoplasmosis).

9. ACMSF. Risk profile in relation to toxoplasma in the food chain, 2012.

10. Defra, PHE. United Kingdom Zoonoses Report (https:// www.gov.uk/government/collections/zoonoses-reports).
11. ONS. Regions (former GORs) (http://webarchive. nationalarchives.gov.uk/20160105160709/http://www. ons.gov.uk/ons/guide-method/geography/beginner-s-guide/ administrative/england/government-office-regions/index. html). Accessed 25 April 2016.

12. Cook A, et al. Sources of toxoplasma infection in pregnant women: European multicentre case-control study. European Research Network on Congenital Toxoplasmosis. British Medical Journal 2000; 321: 142-147.

13. Buffolano W, et al. Risk factors for recent toxoplasma infection in pregnant women in Naples. Epidemiology \& Infection 1996; 116: 347-351.

14. Baril L, et al. Risk factors for Toxoplasma infection in pregnancy: a case-control study in France. Scandinavin Journal of Infectious Diseases 1999; 31: 305-309.

15. Nash $\mathbf{J}$, et al. Risk factors for toxoplasmosis in pregnant women in Kent, United Kingdom. Epidemiology \& Infection 2005; 133: 475-483.

16. Said B, et al. Hepatitis E outbreak on cruise ship. Emerging Infectious Diseases 2009; 15: 1738-1744.

17. Said B, et al. Hepatitis E virus in England and Wales: indigenous infection is associated with the consumption of processed pork products. Epidemiology \& Infection 2014; 142: 1467-1475.

18. Scallan E, et al. An assessment of the human health impact of seven leading foodborne pathogensin the United States using disability adjusted life years. Epidemiology \& Infection 2015; 143: 2795-2804.

19. Mangen M, et al. Cost-of-illness and disease burden of food-related pathogens in the Netherlands, 2011. International Journal of Food Microbiology 2015; 196: 84-93. 